\title{
REVIEW ARTICLE \\ The development of the social brain in human infancy
}

\author{
Tobias Grossmann and Mark H. Johnson \\ Centre for Brain and Cognitive Development, School of Psychology, The Henry Wellcome Building, Torrington Square, Birkbeck \\ College, London WC1E 7HX, UK
}

Keywords: development, EEG, infancy, social brain, social cognition

\begin{abstract}
Much research has focused on how the adult human brain processes the social world, yet until recently little was known about the early development of these abilities. Developmental studies inform debates about the specificity of social functions in the adult cortex. This review highlights recent work, mainly based on electroencephalography/event-related potential methods, examining the precursors of the human social brain network during infancy in several domains such as face and eye gaze processing, the perception of emotions, decoding biological motion, perceiving human actions and joint attention. The findings illustrate that the human brain is fundamentally adapted to develop within a social context, and that this context contributes to many of the specializations seen in the adult cortex.
\end{abstract}

\section{Introduction}

Humans, like other primates, are intensely social creatures. One major function of our cognitive apparatus is to enable us to recognize, manipulate and behave with respect to socially relevant information. The field of social cognition attempts to understand and explain how thoughts, feelings and behaviour of individuals are influenced by the presence of, and through interaction with, others (Allport, 1985; Fiske, 1995).

There is evidence for the relative independence of social cognition from other aspects of cognition. For example, individuals with either frontal or prefrontal cortex damage show impaired social behaviour and functioning, despite the retention of some intact cognitive skills such as memory and language (Anderson et al., 1999; Blair \& Cipolotti, 2000; Fine et al., 2001). The fact that social cognition can be relatively impaired after such an injury while sparing aspects of non-social cognition suggests that unique neural circuits may contribute to social cognition. A similar dissociation between social cognition and non-social cognitive skills is often observed in persons with prosopagnosia, who show selective impairments in the perceptions of faces but preserved perception for non-social stimuli (Kanwisher, 2000). Furthermore, it has been shown that social and non-social behaviours are both highly heritable and yet largely independent, with most of the genetic influence being specific to either social or non-social behaviours (Ronald et al., 2005). More evidence for the partial independence of social cognition is gleaned from studies of persons with Williams' syndrome or autism. Individuals with Williams' syndrome appear to have relatively preserved basic social cognitive skills (i.e. facial processing and simple abilities regarding a theory of mind), despite having deficits in spatial cognition (Karmiloff-Smith et al., 1995; Bellugi et al., 2000). This partial preservation of social cognition is in direct

Correspondence: Dr T. Grossmann, as above.

E-mail: t.grossmann@bbk.ac.uk

Received 27 October 2006, revised 7 December 2006, accepted 1 January 2007 contrast to persons with high-functioning autism and Asperger's syndrome, who show impairments in social cognition and social behaviour that may not be related to general cognitive abilities (Heavey et al., 2000; Klin, 2000).

Together, these findings lend support to the hypothesis that there is a network of specific brain areas preferentially involved in the processing of social information. This network has been referred to as the 'social brain' (Adolphs, 1999, 2001, 2003). This social brain hypothesis has also been maintained and influenced by the thinking in the area of evolutionary anthropology (Brothers, 1990). To explain primates' and particularly humans' unusually large brains, it has even been claimed that the computational demands of living in complex social groups selected for increases in neocortex - a view put forward as the social brain hypothesis (Dunbar, 2003) or the Machiavellian intelligence hypothesis (Whiten \& Byrne, 1997).

A new discipline termed 'social cognitive neuroscience' has emerged that investigates the neural underpinnings of human social behaviour. Its agenda has been described in terms of seeking 'to understand phenomena in terms of interactions between three levels of analysis: the social level, which is concerned with the motivational and social factors that influence behaviour and experience; the cognitive level, which is concerned with the information-processing mechanisms that give rise to social-level phenomena; and the neural level, which is concerned with the brain mechanisms that instantiate cognitive-level processes' (Ochsner \& Lieberman, 2001: p. 717). The majority of the research activities in this area have been focused on how adults' brains make sense of the social world (for reviews, see Adolphs, 1999, 2001, 2003; Frith \& Frith, 1999, 2006; Blakemore \& Frith, 2004; Beer \& Ochsner, 2006; Lieberman, 2006). This provides us with important insights into the full-fledged, fully developed neural machinery that deals with very complex social problems. However, from an ontogenetic perspective, the following questions arise: how do these sophisticated capacities of the brain in reading others' social behaviour develop, and what are the critical developmental precursors of these adult abilities? 
The answers to these questions are important for a variety of reasons. An understanding of the early emergence of the social brain will be of relevance for social, educational and clinical policies (Spitzer, 2002; Blakemore \& Frith, 2005). Furthermore, a developmental perspective may shed light on debates within adult cognitive neuroscience such as that concerning the specificity of face processing areas of cortex (Gauthier et al., 1999; Kanwisher, 2000).

The earliest stage of postnatal development, infancy (0-2 years), is the time of life during which enormous changes take place - the 'helpless' newborn seems almost a different creature from the active, inquisitive 2-year-old. During this period, human infants develop in a world filled with other people, including parents, siblings, other family members, friends and strangers. Relating socially to others has not only profound effects on what they feel, think and do, but is also essential for their healthy development and for optimal functioning throughout life. Indeed, the ability to learn from other humans is perhaps one of the most important adaptations of our species (Csibra \& Gergely, 2006). Therefore, developing an understanding of other people is one of the most fundamental tasks infants face in learning about the world. The development of the brain circuitries involved in all kinds of cognitive processes depend upon the interaction of two broad factors: nature (our inheritence or genetic factors) and nurture (environmental influences). If we aim to understand how these factors interact to 'build' the mature social brain network, it seems of particular importance to look at how the human brain deals with social information during infancy.

The ontogeny of the social brain network is one aspect of human postnatal functional brain development, and it is therefore useful to consider work within this field within the context of three general perspectives on developing brain function. Johnson (2001) presented three distinct, but not necessarily mutually exclusive, frameworks for understanding postnatal development in humans. According to a 'maturational' perspective, functional brain development involves the sequential coming 'on line' of a number of modular cortical regions. The maturation of a given region is thought to allow or enable advances in the perceptual, cognitive or motor abilities of the child. As applied to the neurodevelopment of social cognition, this implies that more complex aspects (such as 'theory of mind' computations) will be dependent on the maturation of associated cortical regions (possibly within the prefrontal cortex). The second perspective, 'skill learning', argues for parallels between the dynamic brain events associated with the acquisition of complex perceptual and motor skills in adults, and the changes in brain function seen as infants and children come to perform simpler tasks successfully. From this perspective, it has been argued that some cortical regions become recruited for processing social information because typical humans become perceptual experts in this domain (Gauthier \& Nelson, 2001). A third perspective, 'interactive specialization', posits that functional brain development, at least in the cerebral cortex, involves a process of specialization in which regions go from initially having very broadly tuned functions to having increasingly finely tuned (more specialized) functions (Johnson, 2001). A consequence of increased specialization of cortical regions is the increasingly focal patterns of cortical activation resulting from a given task demand or stimulus. By this view, some regions of the cortex gradually become increasingly specialized for processing social stimuli and thus become recruited into the social brain network.

The purpose of this review is to bring together and discuss the work that has been done in recent years to examine the cognitive neuroscience of social perception in infancy. The underlying motivation for reviewing the current state of affairs is two-fold: first, it is meant to encourage the crosstalk between disciplines, particularly between those researchers studying the adult social brain and those trying to understand its early development; second, at this early stage with the field still uncertain on its feet, it seems worthwhile to contemplate where we are headed. To this end, we will focus on the emerging evidence available on infants' neural correlates of processing social information. The following sections will present and discuss findings from several areas of processing social information in infancy: face and gaze processing, perception of emotions, decoding biological motion, perceiving human actions and joint attention. These findings are mainly based on electroencephalography (EEG)/eventrelated potential (ERP) methods, which are the most commonly used methods with this age group (for recent reviews concerning infant structural brain development, see Johnson, 2005a; for how this relates to EEG/ERP measures, see Csibra et al., in press). Based on the evidence presented, we will formulate future research questions, identify promising methodological developments and propose new directions for the field. We do not cover speech perception and language development, as this aspect of social brain development in infants has recently been reviewed (e.g. Kuhl, 2004).

\section{Face processing}

The face provides a wealth of socially relevant information. To detect and recognize faces is therefore commonly considered to be an important adaptation of social animals. From birth, human infants preferentially attend to some face-like patterns (Morton \& Johnson, 1991; Johnson, 2005b), suggesting a powerful mechanism to bias the input that is processed by the newborn's brain. Besides the detection of faces, another important aspect is to recognize familiar faces. Newborns first recognize their mother's face on the basis of information from the outer contour of the head, hairline, and the internal configuration of eyes, nose and mouth (Bushnell et al., 1989). But it is not until 6 weeks of life that they become able to do this recognition solely on the face's internal configuration (de Schonen \& Mathivet, 1990). The face preference observed in newborns is thought to be guided largely by subcortical brain structures, while it has been proposed that the maturation of visual cortical areas is necessary for the emergence of the more sophisticated competencies underlying identity recognition from faces (for a discussion, see Johnson, 2005b).

Investigations of brain areas involved in face processing in infants have been limited by ethical and technical issues (de Haan \& Thomas, 2002). One exception has been a study by Tzourio-Mazoyer and colleagues, who took advantage of the opportunity to perform positron emission tomography (PET) on infants in an intensive care unit as part of a clinical follow-up (Tzourio-Mazoyer et al., 2002). In this smallscale study, a group of six 2-month-olds were imaged while they watched a face or an array of coloured diodes used as a control stimulus. A subtraction analysis revealed that faces activated a network of areas in 2-month-old infants' brains similar to that described as the core system for face processing in adults (Haxby et al., 2000). More specifically, a significant activation was reported in an area in infants' right inferior temporal gyrus, which is thought to be the homologue of the adult fusiform face area (Gauthier et al., 1999; Kanwisher, 2000). It is interesting to note that a cortical region that at the age of 2 months is neuroanatomically immature (Huttenlocher \& Dabholkar, 1997; Huttenlocher, 2002) and has only a low level of metabolic activity (Chugani \& Phelps, 1986; Chugani et al., 1987) can exhibit functional activity. In addition, face perception activated bilateral inferior occipital and right inferior parietal areas in infants. The former has been implicated in early perceptual analysis of facial features, whereas the latter is thought to support spatially directed attention in adults (Haxby et al., 2000). Interestingly, and contrary to what is known from adults, face processing in 2-month-olds also 
recruited areas in the inferior frontal and superior temporal gyrus, which have been identified as a part of the adult language network. One possible interpretation is that the co-activation of face and future language networks has a facilitatory effect on social interactions guiding language learning by looking at the speaker's face (TzourioMazoyer et al., 2002).

While bearing in mind the small sample size and non-optimal control stimulus, the study by Tzourio-Mazoyer and colleagues provided important information by identifying the neural substrates of the infant face processing network. However, as mentioned earlier, due to ethical and technical concerns with the use of neuroimaging methods like PET and functional magnetic resonance imaging (fMRI) in infants, evidence coming from these kinds of studies is rare. Therefore, most studies that have examined infant brain function rely on the more readily applicable recording of EEG measures, which provide an excellent temporal resolution but are relatively poor in their spatial resolution.

We now discuss briefly the empirical evidence available on infants' face processing using ERPs (for a more detailed review, see de Haan et al., 2003). In adults, human faces elicit an N170 response, which is most prominent over posterior temporal sites and is larger in amplitude and longer in latency to inverted than to upright faces (Bentin et al., 1996; de Haan et al., 2002). This component is not modulated by the inversion of monkey faces (de Haan et al., 2002), nor when upright objects are compared with inverted objects (Bentin et al., 1996). This selective inversion effect for human faces has been taken as evidence for a face-related processing mechanism generating the N170.

On the basis of waveform morphology and some of its response properties, it has been suggested that the infant N290 is a precursor to the adult N170. In these studies, infants' and adults' ERPs were measured in response to upright and inverted human and monkey faces (de Haan et al., 2002; Halit et al., 2003). The infant N290 is a negative-going deflection observed over posterior electrodes whose peak latency decreases from $350 \mathrm{~ms}$ at 3 months to $290 \mathrm{~ms}$ at 12 months of age (Halit et al., 2003). This is consistent with the latency of many prominent ERP components reducing with increasing age during childhood. The results of these studies indicate that at 12 months of age the amplitude of the infant N290, like the adult $\mathrm{N} 170$, increases to inverted human but not inverted monkey faces when compared with the upright faces. However, the amplitude of the N290 was not affected by stimulus inversion at an earlier age ( 3 and 6 months).

By applying new source separation and localization methods (Richards, 2004) to the infant ERP data (Halit et al., 2003) it was possible to identify candidate cortical sources of the face inversion effect. This analysis suggested that generators in the left and right lateral occipital area, the right fusiform face area, and the right superior temporal sulcus (STS) discriminated between upright and inverted faces in 3- and 12-month-olds (Johnson et al., 2005). All three cortical areas have been implicated in the core face processing system in adults (Haxby et al., 2000), and they also generally overlap with the areas identified in the previously discussed PET study with 2-month-olds (Tzourio-Mazoyer et al., 2002).

It is important to point out that the development of the brain processes reflected in the N170/N290 continues well beyond infancy (for a review, see Taylor et al., 2004). Whereas latency of the adult N170 is delayed by face inversion, no such effect is observed for the latency of the infant N290 at any age (de Haan et al., 2002; Halit et al., 2003). There is evidence that suggests that this latency effect is not apparent until 8-11 years (Taylor et al., 2004). Another important developmental finding is that while the amplitude of the adult N170 is larger to the monkey faces, infants' N290 shows the opposite pattern.
A completely adult-like modulation of the amplitude of the N170 has not been reported until 13-14 years (Taylor et al., 2004).

To date, face processing, besides speech perception (DehaeneLambertz et al., 2002; Kuhl, 2004), represents the best studied aspect of the infant social brain. The evidence available from PET and EEG/ERP studies suggests that most of the brain areas and mechanisms implicated in adult face processing can be activated within the first few months of postnatal life. However, there are some differences, such as the activation of the inferior frontal and superior temporal gyrus in 2-month-olds (Tzourio-Mazoyer et al., 2002), and the STS generator of the inversion effect found in 3- and 12-montholds (Johnson et al., 2005), that do not directly map onto the mature face processing system. In addition to the extra regions involved while infants perceive faces, another important observation in the infant ERP work is that the infant face processing system possesses much broader response properties that are not yet as finely tuned to upright human faces. This suggests that despite the gradual cortical specialization seen throughout the first year of life, the system continues to specialize well beyond infancy. These changes in the degree of specialization of processing, and the spatial extent of cortical activation, are in accordance with the interactive specialization perspective alluded to earlier (Johnson, 2001). A pattern of increasingly focal activation in response to faces is further supported by several recent fMRI studies comparing children and adults indicating that faces resulted in a more distributed pattern of activation in 8- to 12-year-old children than in adults (Passarotti et al., 2003; Aylward et al., 2005).

\section{Eye gaze processing}

An important social signal encoded in faces is eye gaze. The detection and monitoring of eye gaze direction is essential for effective social learning and communication among humans (Bloom, 2000; Csibra \& Gergely, 2006). Eye gaze provides information about the target of another person's attention and expression, and it also conveys information about communicative intentions and future behaviour (Baron-Cohen, 1995). Eye contact is considered to be the most powerful mode of establishing a communicative link between humans (Kampe et al., 2003). From birth, human infants are sensitive to another person's gaze as reflected in their preference to look at faces that have their eyes open rather than closed (Batki et al., 2000). Furthermore, newborns look longer at faces with direct/mutual gaze as compared with faces with averted gaze (Farroni et al., 2002). Eye gaze has also been shown to cue effectively newborn infants' attention to spatial locations, suggesting a rudimentary form of gaze following Farroni et al. (2004b). The question that arises is how the behaviourally expressed preference for mutual gaze and the capacity to follow gaze are implemented in the infant brain.

By 4 months, the infant brain manifests enhanced processing of faces with direct gaze as indexed by an increased amplitude of the N290 than with averted gaze (Farroni et al., 2002). This finding is obtained even when the head is averted but direct mutual gaze is maintained (Farroni et al., 2004a). However, enhanced neural processing to faces with direct gaze is only found when eyes are presented in the context of an upright face.

Furthermore, source separation and localization methods were used to identify the cortical sources of the scalp-recorded ERP effects sensitive to eye gaze (Johnson et al., 2005). Contrary to adults, who show specific activations associated with eye gaze perception in the STS (Allison et al., 2000), cortical generators localized in the fusiform gyrus discriminated gaze direction best in infants. Although it has been shown that the amplitude of the N290 in infants is modulated by eye gaze (Farroni et al., 2002, 2004b) and face orientation (de Haan 
et al., 2002; Halit et al., 2003), the amplitude of the adult N170 is only affected by face inversion but not by direction of gaze (Taylor et al., 2001; Grice et al., 2005). Taken together, the difference in the response properties of the infant N290 vs. the adult N170 suggest that face and eye gaze share common patterns of cortical activation early in ontogeny, which later partially dissociate and become more specialized, a developmental change that is consistent with 'interactive specialization' (Johnson, 2001).

Another communicative function of eye gaze is to direct attention to certain locations, events and objects. Understanding the relations between eye gaze and target objects is particularly important for aspects of development such as word learning. Comprehending that another's gaze direction refers to a specific object allows the child to associate the object with a name or emotional expression (Baldwin \& Moses, 1996). Adults' gaze has been found to facilitate object processing at a neural level in infants as young as 4 months (Reid et al., 2004). In their ERP study, objects that were previously cued by eye gaze elicited a diminished positive slow wave observed between 700 and $1000 \mathrm{~ms}$ over right fronto-temporal channels. A diminished positive slow wave is thought to indicate deeper memory encoding (Nelson \& Collins, 1991). This suggests that eye gaze as a social cue facilitates brain processes involved in memory encoding that might assist infants' learning.

In another ERP study, 9-month-old infants and adults watched a face whose gaze shifted either towards (object-congruent) or away from (object-incongruent) the location of a previously presented object (Senju et al., in press). This paradigm was based on that used in an earlier fMRI study (Pelphrey et al., 2003) and was designed to reveal the neural basis of 'referential' gaze perception. When the ERPs elicited by object-incongruent gaze shifts were compared with the object-congruent gaze shifts, an enhanced negativity around $300 \mathrm{~ms}$ over occipito-temporal electrodes was observed in both infants and adults. This suggests that infants encode referential information of gaze using similar neural mechanisms to those engaged in adults. However, only infants showed a fronto-central negative component that was larger in amplitude for object-congruent gaze shifts. It is thus possible that in the less specialized infant brain, the referential information of gaze is encoded in broader cortical circuits than in the more specialized adult brain.

In summary, infants show specific modulations of cortical responses associated with eye gaze perception, which differ from what is seen in adults. Nevertheless, the findings reviewed also suggest that from an early age they successfully discriminate between direct and averted gaze, and can make use of gaze cues when encoding objects in memory. This indicates that, although the cortical structures involved in the perception of gaze direction may not be fully differentiated in infancy, they are at least partially functional. This may be critical for generating social communication and learning from others.

\section{Perception of emotions}

Social behaviour is coupled with emotion. During social interactions, emotions are overtly expressed by the infant, whereas displays of emotion are normally more regulated in adults. Nevertheless, most brain structures that have been shown to be important in processing emotions in adults have also turned out to be essential for social behaviour (for a review, see Adolphs, 2003). Detecting emotion in others through their facial expressions is an important way to communicate emotions in social interactions (Izard, 1991). Recognizing facial expressions permits us to detect another person's emotional state and provides cues on how to respond in these social interactions.
In an ERP study examining infants' processing of facial expressions (Nelson \& de Haan, 1996), 7-month-olds watched happy vs. fearful faces in a first experiment. Results revealed that fearful faces elicited an enhanced negative component (Nc) peaking around $500 \mathrm{~ms}$. The $\mathrm{Nc}$ has its maximum at frontal and central sites and has been thought of as an obligatory attentional response sensitive to stimulus familiarity (Courchesne et al., 1981; Snyder et al., 2002). Dipole modelling has revealed that the cortical sources of the $\mathrm{Nc}$ can be localized in the anterior cingulate and other prefrontal regions (Reynolds \& Richards, 2005). This suggests that 7-month-old infants in Nelson and de Haan's study allocated more attentional processing resources to the unfamiliar fearful than to the familiar happy expression as indicated by an enhanced Nc. In a second experiment Nelson and de Haan showed another group of 7-month-olds fearful vs. angry faces but found no difference in their ERP response. One possibility is that infants in Nelson \& de Haan's (1996) second experiment, although able to discriminate between the angry and fearful expressions (Serrano et al., 1995), did not display different brain responses to the two expressions because they perceived the signal value of both expressions as 'negative'. Another plausible explanation is that infants perceived both expressions as equally unfamiliar and therefore the Nc, which is sensitive to the familiarity of a stimulus, did not differ between conditions (for a further discussion of how the neural processing of angry in contrast to fearful facial expressions develops during infancy, see Grossman et al., in press). It is also important to note that in adults differences in the processing of fearful and angry facial expressions studied with fMRI were observed in subcortical structures such as amygdala (Whalen et al., 2001), and therefore ERP measures used with infants might not be sensitive to differences evoked on the subcortical level.

Processing of facial expressions has been studied as a function of particular experiences such as early institutional rearing (Parker et al., 2005) and maternal personality (de Haan et al., 2004). In general, these studies suggest that experiential factors influence the ways that infants process facial expressions. It is important to note that the reverse may also be true, i.e. that neural development occurring at the end of the first year (Diamond, 1991, 2000; Johnson, 2001, 2005a) may impact infant behaviour and subsequently infants' experiences with others. Although the control of infants' orientating is partly in the hands of the caregivers' presentation of relevant information, the infant is clearly involved in soliciting attention and information from adults (Stern, 1985; Baldwin \& Moses, 1996). With the development of the anterior attention system during the first year of postnatal life, more direct control of attention passes from the caregiver to the infant (Bush et al., 2000; Posner \& Rothbart, 2000), which might influence and regulate infants' emotional exchanges and experiences.

Emotion produces not only characteristic changes in facial patterning but also in respiration, phonation and articulation, which in turn determine the acoustic signal (Scherer, 1989). Thus, speech melody, also referred to as emotional prosody, serves as a signal for various emotions. Adult listeners across cultures can reliably and readily recognize different emotions on the basis of vocal cues (Banse \& Scherer, 1996; Scherer et al., 2001) and do so using some of the same brain structures used to recognize facial expressions (Adolphs et al., 2002). How the processing of emotional prosody develops over the course of ontogeny is only poorly understood. Unlike the extensive behavioural work on processing facial expressions in infancy, studies on infants' perception of vocal expressions of emotion are relatively scarce (Walker-Andrews, 1997).

In a recent ERP study 7-month-old infants' processing of emotional prosody was examined using ERPs (Grossmann et al., 2005). In their study, infants heard semantically neutral words that were spoken with 
either a happy, angry or neutral voice. Angry prosody elicited a negative component peaking around $450 \mathrm{~ms}$ over fronto-central sites in infants' ERPs, suggesting greater allocation of attention to the angry prosody. This is consistent with the notion of an evolutionarily driven propensity to react more strongly to negative than to positive or neutral stimuli (Cacioppo \& Berntson, 1999). A positive slow wave (700-1000 ms) was elicited by angry and happy prosody over temporal electrode sites, whereas words with neutral prosody returned to baseline. This indicates an enhanced processing in auditory (temporal) brain structures of the emotionally loaded stimuli (happy and angry), which is in line with recent fMRI work on adults' processing of emotional speech (Mitchell et al., 2003; Grandjean et al., 2005). These findings suggest that very early in development, the human brain detects emotionally loaded words and shows differential attentional responses depending on their emotional valence.

The way that emotions are perceived when communicated either by the face or the voice has been studied in each modality separately. However, in most social interactions, emotional information is communicated simultaneously by different modalities such as the face and voice. Thus, the question arises: how is emotional information from the face and voice integrated? To address this question, processing of emotionally congruent and incongruent facevoice pairs has been studied using ERP measures (Grossmann et al., 2006). In that study, infants watched facial expressions (happy or angry) and, after a delay of $400 \mathrm{~ms}$, heard a word spoken with a prosody that was either emotionally congruent or incongruent with the facial expression being presented. Emotionally congruent face-voice pairs elicited similar ERP effects as recognized items in previous memory studies with infants, children and adults (Friedman, 1991; Friedman et al., 1992; Rugg \& Coles, 1995; Nelson et al., 1998). This provides electrophysiological evidence that 7-month-olds integrate emotional information across modalities and recognize common affect in the face and voice, a finding which is in line with previous behavioural studies (Soken \& Pick, 1992; Walker, 1982; WalkerAndrews, 1986).

As the face-voice pairs presented to the infants were novel to them, these findings not only indicate that infants recognized common affect but, moreover, that they applied their knowledge about emotions in face and voice to draw inferences about what might be appropriate emotional face-voice pairings. Extending previous behavioural findings, the ERP data revealed insights into the time course and characteristics of the processes underlying the integration of emotional information from face and voice in the infant brain. An emotionally incongruent prosody elicited a larger fronto-central negative component $(400-600 \mathrm{~ms})$ in infants' ERPs than did an emotionally congruent prosody, indicating increased allocation of attention. Conversely, the amplitude of infants' positive component (600$1000 \mathrm{~ms}$ ), with a maximum over parietal channels, was larger to emotionally congruent than to incongruent prosody, indexing recognition of the congruity between face and voice (Grossmann et al., 2006).

Very little is currently known about how eye contact influences the processing of emotional expressions in the infant brain. It has been previously argued that direct gaze is probably involved in the communication of increased emotional intensity, regardless of the particular emotion being expressed (Kimble \& Olszewski, 1980; Kleinke, 1986). Despite the intuitive appeal of the long-standing notion that direct gaze may facilitate emotional expression processing, recent brain and behavioural research with adults has shown that the way in which gaze direction influences emotion perception depends on the emotion in question (Adams \& Kleck, 2003, 2005; Adams et al.,
2003). For example, averted gaze enhanced the perception of fear faces whereas direct gaze enhanced the perception of angry faces (Adams \& Kleck, 2005).

The question of how eye gaze interacts with emotional expression has also been examined in young infants using ERP measures (Striano et al., 2006a). In their study, 4-month-old infants were presented with neutral, happy and angry facial expressions when accompanied with direct and averted gaze. The results show that neural processing of angry faces is enhanced in the context of direct gaze, whereas there is no effect of gaze on the processing of the other expressions. This neural sensitivity to the direction of angry gaze can be explained in two different ways. First, infants at this age who have very rarely seen angry faces (Campos et al., 2000) might be primed to detect novel/unfamiliar emotions that are directed toward them. Second, it is also possible that infants detect the potential threat conveyed by an angry face with gaze directed at them, which is reflected in an enhanced neural response. To find out which explanation ('novelty' vs. 'threat') can account for these ERP findings, it would be necessary to examine the processing of a facial expression that is equally unfamiliar to the infant but whose perception is enhanced in the context of averted gaze; one such emotion is fear (Adams et al., 2003; Adams \& Kleck, 2005). Nevertheless, it is important to note that by using ERP measures it was possible to show that the neural processing of facial expression is influenced by the direction of eye gaze.

The investigation of the electrophysiological correlates of perceiving facial, vocal and bimodal emotional cues has provided important insights into the brain mechanisms guiding infants' emerging understanding of emotional expressions. Interestingly, the body of evidence presented here shows that effects of emotion, in contrast to the 'early' effects elicited by face and gaze manipulations alone, are reflected in specific modulations of components that occur relatively 'late' in the infants' ERP ( $>300 \mathrm{~ms}$; by contrast, some emotional expressions can have effects at very short-latency ERP components in adults, Eimer \& Holmes, 2002). This might be related to the fact that learning to identify an emotion requires more complex cognitive and brain operations, which integrate various perceptual elements provided by face and (or) voice, and retrieve specific information about the emotion stored in memory. The studies that have been conducted so far have only looked at a limited set of emotions (happiness, fear and anger). This work should be extended to other basic (disgust, sadness, surprise) and complex (social) emotions in order to understand the emotion-specificity of the effects summarized here.

\section{Decoding biological motion}

Another issue that is vital to a social species is how to identify biological agents and in particular conspecifics. The detection and interpretation of human-specific biological motion is thought to be a critical process towards this identification. Behavioural research using point-light displays (PLDs; Johansson, 1973) suggests that very early on human infants detect biological motion and discriminate it from other forms of motion (e.g. drifting dots), and they show a specific preference for upright human movement (Bertenthal, 1993). The basis for this orientation preference may be the characteristic motion patterns of the feet (Troje \& Westhoff, 2006).

In an ERP study, 8-month-old infants' processing of upright and inverted PLDs depicting human movement was examined (Reid et al., 2006). Inverted compared with upright human motion elicited a greater right posterior N290 in infants' ERPs. These results suggest that 8-month-olds process upright and inverted human motion differently, providing corroborating evidence to what is already known from the behavioural work. More importantly, both inverted 
compared with upright human motion (Reid et al., 2006) and inverted human faces compared with upright human faces (Halit et al., 2003) have been found to elicit a greater N290. This similarity observed in infants' human face and human motion processing suggests that in infants the cortical processes underlying the N290 might be more generally tuned to 'humanness' of a visual stimulus perhaps driven by familiarity, independent of whether the stimulus is a face or a PLD. Furthermore, in another ERP study, 8-month-olds were found to show a greater N290 over right posterior sites to human motion when compared with scrambled motion (Hirai \& Hiraki, 2005). It is interesting to note that a similar amplitude modulation of the infant N290 was observed when human faces were compared with matched visual noise (Halit et al., 2004), which further supports the parallels seen between face and motion processing.

Despite the observed commonalities between face and biological motion processing observed on the N290, it is equally important to point out the differences between the two. Namely, in both studies on biological motion processing, a clear lateralization of the effects to the right hemisphere has been reported (Hirai \& Hiraki, 2005; Reid et al., 2006), whereas no clear lateralization effects have been observed in the studies examining infants' face processing (de Haan et al., 2002; Halit et al., 2003). In adults, both biological motion and face processing have been shown to rely more on cortical activity in the right hemisphere (de Haan et al., 2002; Hirai \& Hiraki, 2005). This suggests that, from the first few months, the infant brain shows a more adult-like pattern of processing for biological motion than for faces.

\section{Perceiving the actions of others}

Human actions are an exceedingly complex set of social stimuli providing dynamic and continuous information about conspecifics' behaviour. The capacity to parse ongoing actions into segments is critical for the interpretation of others' behaviour. There is evidence to suggest that in adults and infants, this parsing of action sequences is partly based upon understanding the goal of an action (Baldwin et al., 2001; Blakemore \& Decety, 2001). In a recent study (Reid et al., in press), 8-month-old infants were presented with video clips showing complete and incomplete goal-directed actions (pouring liquid from a bottle into a glass). In a first behavioural experiment, it was found that infants look longer at the incomplete than at the complete action, indicating a discrimination of the two actions with an increased attention to the incomplete action. In a second experiment, the neural correlates of perceiving complete and incomplete actions were examined by measuring electrophysiological activity and analysing induced gamma oscillatory brain activity (for a detailed methodological review, see Csibra et al., in press). Gamma oscillatory activity is thought to play a fundamental role in binding perceptual information to form a unified percept (Tallon-Baudry \& Bertrand, 1999; Csibra et al., 2000), and it has also been shown to be influenced by top-down attentional processing (Keil et al., 2001). The analysis of 8-month-old infants' gamma oscillatory activity revealed a significant difference between complete and incomplete actions over left frontal channels (216-284 ms) with an increase of gamma activity in the incomplete condition (Reid et al., in press).

There are two ways of interpreting these findings. The first is that increased gamma activity in the incomplete condition indexes a greater allocation of attention to this action sequence. This view is supported by the findings that infants look longer to the incomplete action in the behavioural experiment and that adults show increased gamma activity induced by greater attentional demands (Müller et al., 2000). It is also possible that the frontal gamma oscillatory activity is related to a mismatch detection between anticipated and perceived actions such that it is increased in the case where an action is terminated before a goal is achieved. This interpretation is based on a phenomenon called forward-mapping, suggesting that when infants and adults observe human actions they continuously predict possible motion trajectories and future actions based on their own motor representations (see, for example, Falck-Ytter et al., 2006).

Along these lines, researchers have asked the question whether infants come to understand other people's actions through a mirror neuron system (Rizzolatti \& Craighero, 2004) that maps an observed action onto infants' own motor representation of that action. According to the mirror neuron view, infants are not expected to predict others' action goals before they can perform the action themselves. In a behavioural study (Falck-Ytter et al., 2006), 12-month-olds but not 6-month-olds were found to show proactive (anticipatory) goal-directed eye movements during observation of an action (putting objects into a box). Because it is known that infants do not master this kind of motor task until 7-9 months of age (Bruner, 1973), this finding was taken as evidence for the mirror neuron view of action understanding. Furthermore, based on the finding that proactive eye movements were only found when a human agent produced the action but not when the objects were self-propelled (Falck-Ytter et al., 2006), it was argued that a human agent is required to activate the mirror neuron system. These findings are also interesting in light of evidence showing that good motor skills in 8-month-old infants correlate with their sensitivity to discriminate biologically possible from impossible arm movements (Reid et al., 2005). Despite the value of these interesting behavioural findings, a shortcoming is that they provide only indirect evidence for neurallevel explanations. Therefore, conclusions about the underlying neural mechanisms are limited and need further assessment using neuroimaging techniques.

Taken together, it is obvious that more research is needed to test the developmental aspects of existing theories of action understanding in general and simulation theories based on the mirror neuron system in particular (see Csibra, in press). A candidate neural signature that might be useful for the study of action perception is the mu wave (oscillatory bursts at 9-11 Hz), which can be measured with EEG. This wave activity appears to be associated with the motor cortex, and is diminished with movement or an intent to move, or when others are observed performing actions (Oberman et al., 2005). Therefore, EEG oscillations in the mu wave range over sensorimotor cortex are thought to reflect mirror neuron activity, and research is underway to investigate mu wave activity in infants in relation to action perception (Nystroem et al., 2006).

\section{Joint attention}

One of the major developmental changes in infants' engagement with others is the transition from participating in dyadic (face-to-face) interactions to developing a capacity to engage in triadic (infant-otherobject) joint attention exchanges. Besides attending to an external object or event him/herself, the ability to attend jointly with another person requires the infant to monitor (1) the other person's attention in relation to the self and (2) the other person's attention toward the same object or event. Triadic relations between two minds and an object are thought to be uniquely human representations (Baron-Cohen, 1995; Tomasello et al., 2005), supporting shared attention and collaborative goals. The dorsal part of the medial prefrontal cortex has been identified as the neural substrate supporting these kinds of representations in the adult human brain (Frith \& Frith, 2006; Saxe, 2006). It has been shown that already by 3 months of age, infants are able to discriminate behaviourally between dyadic and joint attention 
interactions (Striano \& Stahl, 2005). In that study, infants gazed and smiled more when, in the joint attention condition, an experimenter alternated visual attention between the infant and the object than when the experimenter simply looked at the object without engaging/addressing the infant. Despite this early sensitivity to triadic interactions, a more robust understanding of joint attention is not in place until 9 months of age (Tomasello et al., 2005).

Striano et al. (2006b) used a novel interactive paradigm to examine the ERP correlates of joint attention in 9-month-old infants in which an adult interacted live with each infant in two contexts. In the joint attention context the adult looked at the infant and then at the computer screen displaying a novel object, whereas in the non-joint attention context the adult only looked at the novel object presented on the screen. Objects presented in the joint attention context compared with objects in the non-joint attention context were found to elicit a greater Nc peaking around $500 \mathrm{~ms}$ with a maximum over frontal and central channels. The $\mathrm{Nc}$ is generated in the prefrontal cortex and indicates the allocation of attention to a visual stimulus with a greater amplitude indexing more allocation of attentional resources (Reynolds \& Richards, 2005). Therefore, Striano et al. (2006b) suggested that infants benefit from the joint attention interaction and thus devote more attentional resources to those objects presented in this context. Another interesting finding from this study is that the amplitude of the $\mathrm{Nc}$ in both contexts appeared to be substantially larger than in previous ERP studies (de Haan \& Nelson, 1997, 1999). It is possible that rather than viewing the stimuli passively on the screen as in the previous work, the application of a novel interactive paradigm increased the social significance for the infant as reflected in a general increase in amplitude of the Nc.

The acquisition of the ability to co-ordinate attention with a social partner is thought to be critical to infants' active participation in social learning opportunities, which is related to subsequent cognitive, particularly language, development (Bloom, 2000). Hence in another line of research, the relationship between certain neural processes, joint attention and language development has been investigated (Caplan et al., 1993; Mundy et al., 2000; Mundy et al., 2003). In a clinical study, cortical metabolism in infants was measured using PET prior to hemispherectomy for intractable seizure disorder (Caplan et al., 1993). Higher rates of preoperative glucose metabolism in frontal cortical regions, especially left frontal region, was a positive predictor of the postoperatively assessed tendency to initiate joint attention bids. To assess further the role of the frontal cortex, resting EEG coherence and its relation to joint attention skills in typically developing infants was examined (Mundy et al., 2000). EEG coherence is a measure of phase synchrony between spatially separated EEG generators, which provides an index of aspects of neural network integration (Thatcher et al., 1986). EEG coherence measures of left frontal, as well as left and right central cortical activity were found to be associated with the infants' tendency to initiate joint attention at 14 and 18 months. Furthermore, in a followup study, it was shown that measures of joint attention and EEG coherence at 14 months were both related to language development at 24 months (Mundy, Fox, \& Card, 2003).

In summary, there is evidence that joint triadic interaction with the infant indeed has an effect on brain structures, probably localized in the prefrontal cortex, associated with the allocation of attentional processing resources (Striano et al., 2006b). As already seen in previous sections, this provides support for the view that social information has an impact and interacts with attentional processes, which might point to an important mechanism of how social interaction influences information processing early in development. At the outset of this section, based on behavioural findings, we stated that one of the major developmental changes in infants' engagement with others is the transition from participating in dyadic (face-to-face) to triadic (infant-other-object) joint attention exchanges. This behaviourally evident transition has not been examined in the studies summarized here, as infants were only tested at ages at which they already engage successfully in triadic interactions. Thus, in order to elucidate the brain mechanisms underlying behavioural change it seems worthwhile also to test younger infants.

\section{Conclusions}

Various neuroimaging methods (EEG, ERP, PET) have successfully been used to study different aspects of social information processing in infants. The available evidence on the neural processes related to face, gaze, emotion, biological motion, action and joint attention discussed in this review revealed insights into how the infant brain processes information about the social world. However, there are many areas of infant social cognition, such as imitation, social (complex) emotions and 'theory of mind', which remain unexplored (for recent behavioural studies on infant theory of mind, see Onishi \& Baillargeon, 2005; Southgate et al., in press; Surian et al., in press). Future research is needed to examine the neural correlates of these more complex aspects of social cognitive development, and it is very likely that this kind of work will reveal greater differences between adult and infant/child brain function. Regardless, we have seen some similarities between how the adult and infant brains process social information. This suggests relatively early functioning of the cortical structures involved in perceiving other humans. However, this functioning may be broadly tuned, and less specialized, than that seen in adults. Having this network at least partially functional early in life may point to the pivotal role that social communication and learning plays in human development.

Converging evidence indicates that even newborn humans are preferentially attentive to conspecifics, possibly due to subcortical routes for directing attention to social stimuli (Johnson, 2005b). This preferential attention to conspecifics and their interactions will provide the appropriate input for developing cortical circuitry over the first few months. This input, when taken together with other constraints, may ensure that a typical pattern of specialization into a cortical social brain network emerges (Johnson, 2005a,b). According to this view, most parts of the social brain network can be activated in infants, although activation may also extend to other regions not activated under these circumstances in adults. Furthermore, the social brain regions activated may have broader functions (be less finely tuned) than in adults. Some of the evidence consistent with this 'interactive specialization' view is that, compared with adults, infants activate regions in addition to, and surrounding, the core face processing network. Furthermore, face and eye gaze perception have been shown to share common patterns of cortical activation early in ontogeny, which later partially dissociate and become more specialized (Taylor et al., 2001; Farroni et al., 2002, 2004a; Grice et al., 2005; Johnson et al., 2005). These findings support the view that structures in the social brain network initially have more homogeneous response properties, with common processing of many aspects of faces, bodies and actions. With experience, these structures may become more differentiated and specialized in their response properties, finally resulting in the specialized patterns of activation typically observed in adults. This view has implications for atypical development in that some developmental disorders that involve disruption to the social brain network, such as autism, may be characterized in terms of failures or delays of the specialization of structures on the cortical social brain network (for further discussion, see Johnson, 2005b). 
Further progress in infant social cognitive neuroscience crucially depends upon advances on three levels: (1) the development of experimental paradigms that better approximate real life, (2) improvement in neuroimaging methods that suit infants and (3) integration of theories of functional brain development with theories of social cognitive development.

It is experimentally challenging but worthwhile to continue to improve paradigms by modifying the tasks so that they are in accordance with the infant's real world. For example, presenting multimodal stimuli allowed the use of an unusually high number of trials for analysis, suggesting an advantage of multimodal over unimodal stimuli in capturing infants' attention (Grossmann et al., 2006). This is of special interest when the generally low signal-tonoise ratio of infant studies is considered. Moreover, a recent study employed a novel interactive paradigm to assess the neural correlates of joint attention in 9-month-old infants (Striano et al., 2006b), and found that the amplitude of the elicited ERP component was substantially larger in this study than that seen in previous literature, which may be due to the new paradigm employing live interaction. The use of multimodal stimuli and interactive paradigms has the advantage of increased social significance for the child and higher ecological validity as compared with those paradigms utilized in the past. Despite the advantages of using live interaction paradigms, we have to bear in mind that such paradigms also pose certain technical difficulties (e.g. complicated time control, increased motion artefacts).

Infant brain function has been predominantly investigated using EEG and ERPs. Although these methods have helped to understand the neural underpinnings of infant social cognition, they suffer from a relatively poor spatial resolution. Several attempts have been made to localize ERP components to specific anatomical structures in adults (Slotnick et al., 1999). Applying the techniques developed for source localization of adult ERPs to recordings from infants is complicated by two factors. First, these techniques require clean data, which are free from 'noise' (brain activity unrelated to the eliciting event) and which preferably reflect the activation of a single neural source or very few sources. It is very difficult to obtain such clean recordings from human infants, primarily because of the low number of trials in infant studies. Second, source localization techniques depend on assumptions about anatomical structures such as the skull, the cerebrospinal fluid, and the cortical convolutions. The exact physical parameters of these anatomical details are not yet well known for young infants, and applying the parameters used in adult research could lead to mislocalization in infants. However, research is underway using structural MRI to track brain development (Evans, 2006) that might soon provide detailed anatomical information that will help us improve source localization methods with infant data.

Researchers have also begun to use fMRI to localize activity in the infant brain evoked by speech stimuli (Dehaene-Lambertz et al., 2002). Their work revealed that, similar to adults, left-lateralized brain regions (superior temporal and angular gyri) were already active in 3-month-old infants. But fMRI requires rigid stabilization of the head and exposes infants to a noisy acoustic environment. These concerns make it unlikely that fMRI will be routinely used in visual paradigms with infants. A technique called near-infrared spectroscopy that permits the measurement of cerebral haemoglobin oxygenation in response to brain activation has been successfully used to study infant visual, language and memory abilities (for a review, see Aslin \& Mehler, 2005). This novel technique does not suffer from the same problems in its use with infants as fMRI does and might therefore be a promising tool to study the infant social brain. Overall, improving the tools to localize functional activations in the infant brain is critical for our understanding of how the neural structures implicated in the adult social brain develop in early ontogeny.

Even once improved methods show us more clearly how neural events co-vary with infants' processing of social stimuli, the interpretation of such data will remain a major theoretical challenge. In our view, the development of an integrative theoretical framework should rest on three legs: (1) data from adult social cognitive neuroscience, (2) theories of human functional brain development and (3) theories of infant social cognitive development. As outlined above, first attempts have been made to test theories of functional brain development (Johnson, 2001) by examining which parts of what is thought to be the adult social brain network are already active in infants (Johnson et al., 2005). The results of these studies provide preliminary support for the interactive specialization view of human functional brain development, which assumes that developmental changes in the response properties of cortical regions occur as they interact and compete with each other to acquire their functional role. This is in contrast to the 'maturational view' in which the maturation of specific regions of the cortex is related to newly emerging cognitive and social functions, and also in contrast to 'skill learning', which purports that structures on the social brain are engaged by social stimuli due to their role in perceptual expertise. Future work with the imaging methods described above will be necessary to determine which of these viewpoints most accurately reflects the emerging social brain network during the first years.

Another important issue is that although the cross-talk between developmental psychologists and social cognitive neuroscientists has begun on a theoretical level (Decety \& Sommerville, 2003; Meltzoff \& Decety, 2003), there is very little infant brain research that is more directly informed and motivated by already existing theories of infant social cognitive development (Meltzoff, 2002, 2005; Tomasello et al., 2005; Csibra \& Gergely, 2006). The available theoretical frameworks explaining the developmental trajectories of social cognitive capacities provide a rich source of hypotheses that are testable using the neuroimaging tools. In this context it will be of particular importance to identify the neural processes that underlie known social behavioural and social cognitive transitions.

Together, all the challenges listed here underline the truly interdisciplinary nature of the emerging field of social cognitive neuroscience of infancy, and presents the field with the challenge of training scientists who are able to combine knowledge from cognitive neuroscience and developmental psychology in order to shed light on the developing social brain. It is our hope that this review will help to inform and guide future research.

\section{Acknowledgements}

This work was partly supported by the MRC (Programme Grant G97 15587) to M.H.J., and by a Pathfinder grant (CALACEI) from the European Commission. We would like to thank Amrisha Vaish for comments on an earlier version of the manuscript.

\section{Abbreviations}

EEG, electroencephalography; ERPs, event-related potentials; fMRI, functional magnetic resonance imaging; Nc, negative component; PET, positron emission tomography; PLDs, point-light displays; STS, superior temporal sulcus.

\section{References}

Adams, R.B., Gordon, H.L., Baird, A.A., Ambady, N. \& Kleck, R.E. (2003) Effects of gaze on amygdala sensitivity to anger and fear faces. Science, $\mathbf{3 0 0}$, 1536. 
Adams, R.B. \& Kleck, R.E. (2003) Perceived gaze direction and the processing of facial displays of emotion. Psychol. Sci., 14, 644-647.

Adams, R.B. \& Kleck, R.E. (2005) The effects of direct and averted gaze on the perception of facially communicated emotion. Emotion, 5, 3-11.

Adolphs, R. (1999) Social cognition and the human brain. Trends Cogn. Sci., 3, 469-479.

Adolphs, R. (2001) The neurobiology of social cognition. Curr. Opin. Neurobiol., 11, 231-239.

Adolphs, R. (2003) Cognitive neuroscience of human social behaviour. Nature Rev. Neurosci., 4, 165-178.

Adolphs, R., Tranel, D. \& Damasio, H. (2002) Neural systems for recognizing emotion from prosody. Emotion, 2, 23-51.

Allison, T., Puce, A. \& McCarthy, G. (2000) Social perception: role of the STS region. Trends Cogn. Sci., 4, 267-278.

Allport, A. (1985) The historical background of social psychology. In Lindzey, G. \& Aronson, E. (Eds), Handbook of Social Psychology. Random House, New York, pp. 1-46.

Anderson, S.W., Bechara, A., Damasio, H., Tranel, D. \& Damasio, A.R. (1999) Impairment of social and moral behavior related to early damage in human prefrontal cortex. Nature Neurosci., 2, 469-479.

Aslin, R.N. \& Mehler, J. (2005) Near-infrared spectroscopy for functional studies of brain activity in human infants: promise, prospects, and challenges. J. Biomed. Optics, 10, 1-3.

Aylward, E.H., Park, J.E., Field, K.M., Parsons, A.C., Richards, T.L., Cramer, S.C. \& Meltzoff, A.N. (2005) Brain activation during face perception: evidence of a developmental change. J. Cogn. Neurosci., 17, 308-319.

Baldwin, D.A., Baird, J.A., Saylor, M.M. \& Clark, M.A. (2001) Infants parse dynamic action. Child Dev., 72, 708-717.

Baldwin, D.A. \& Moses, L.J. (1996) The ontogeny of social information gathering. Child Dev., 67, 1915-1939.

Banse, R. \& Scherer, K. (1996) Acoustic profiles in vocal emotion expression. J. Pers. Soc. Psychol., 70, 614-636.

Baron-Cohen, S. (1995) Mindblindness: an Essay on Autism and Theory of Mind. MIT Press, Cambridge, MA.

Batki, A., Baron-Cohen, S., Wheelwright, S., Connellan, J. \& Ahluwalia, J. (2000) Is there an innate gaze module? Evidence from human neonates. Infant Behav. Dev., 23, 223-229.

Beer, J. \& Ochsner, K. (2006) Social cognition: a multi level analysis. Brain Res., 1079, 98-105.

Bellugi, U., Lichtenberger, L., Jones, W., Lai, Z. \& St George, M. (2000) The neurocognitive profile of Williams syndrome. J. Cogn. Neurosci., 12, 7-29.

Bentin, S., Allison, T., Puce, A., Perez, A. \& McCarthy, A. (1996) Electrophysiological studies of face perception in humans. J. Cogn. Neurosci., 8, 551-565.

Bertenthal, B.I. (1993) Perception of biomechanical motions by infants: Intrinsic image and knowledge-based constraints. In Granrud, C. (Ed.), Carnegie Symposium on Cognition: Visual Perception and Cognition in Infancy. Erlbaum, Hillsdale, NJ, pp. 175-214.

Blair, R.J.R. \& Cipolotti, L. (2000) Impaired social response reversal: a case of aquired 'sociopathy'. Brain, 123, 1122-1141.

Blakemore, S.J. \& Decety, J. (2001) From the perception of action to the understanding of Intention. Nat. Rev. Neurosci., 2, 561-567.

Blakemore, S.J. \& Frith, U. (2004) How does the brain deal with the social world? Neuroreport, 15, 119-128.

Blakemore, S.J. \& Frith, U. (2005) The Learning Brain: Lessons for Education. Oxford: Blackwell.

Bloom, P. (2000) How Children Learn the Meanings of Words. MIT Press, Cambridge, MA.

Brothers, L. (1990) The social brain: a project for integrating primate behavior and neurophysiology in a new domain. Concepts Neurosci., 1, 27-51.

Bruner, J.S. (1973) Organization of early skilled action. Child Dev., 44, 1-11.

Bush, G., Luu, P. \& Posner, M.I. (2000) Cognitive and emotional influences in anterior cingulate cortex. Trends Cogn. Sci., 4, 215-222.

Bushnell, I.W.R., Sai, F. \& Mullin, J.T. (1989) Neonatal recognition of the mothers face. Br. J. Dev. Psychol., 7, 3-15.

Cacioppo, J.T. \& Berntson, G.G. (1999) The affect system: architecture and operating characteristics. Curr. Dir. Psychol. Sci., 8, 133-137.

Campos, J.J., Anderson, D.I., Barbu-Roth, M.A., Hubbard, E.M., Hertenstein, M.J. \& Witherington, D. (2000) Travel broadens the mind. Infancy, 1, 149219.

Caplan, R., Chugani, H.T., Messa, C., Guthrie, D., Sigman, M., de Traversay, J. \& Mundy, P. (1993) Hemispherectomy for intractable seizures: presurgical cerebral glucose metabolism and post-surgical non-verbal communication. Dev. Med. Child Neurol., 35, 582-592.
Chugani, H.T. \& Phelps, M.E. (1986) Maturational changes in cerebral function in infants determined by 18 FDG positron emission tomography. Science, 231, 840-843.

Chugani, H.T., Phelps, M.E. \& Mazziotta, J.C. (1987) Positron emission tomography study of human brain functional development. Ann. Neurol., 22, 487-497.

Courchesne, E., Ganz, L. \& Norcia, M.E. (1981) Event-related brain potentials to human faces in infants. Child Dev, 52, 804-811.

Csibra, G. (2007) Action mirroring and action interpretation: an alternative account. In Haggard, P., Rosetti, Y. \& Kawato, M. (Eds), Sensorimotor Foundations of Higher Cognition. Attention and Performance XXII. Oxford University Press, Oxford.

Csibra, G., Davis, G., Spratling, M.W. \& Johnson, M.H. (2000) Gamma oscillations and object processing in the infant brain. Science, 290, 15821585.

Csibra, G. \& Gergely, G. (2006) Social learning and social cogniton: the case for pedagogy. In Munakata, Y. \& Johnson, M.H. (Eds), Processes of Change in Brain and Cognitive Development. Attention and Performance XXI. Oxford University Press, Oxford, pp. 249-274.

Csibra, G., Kushnerenko, E. \& Grossmann, T. (in press) Electrophysiological methods in studying infant cognitive development. In Nelson, C.A. \& Luciana, M. (Eds), Handbook of Developmental Cognitive Neuroscience, 2nd edn. MIT Press, Cambridge, MA.

Decety, J. \& Sommerville, J.A. (2003) Shared representations between self and others: a social cognitive neuroscience view. Trends Cogn. Sci., 7, 527-533.

Dehaene-Lambertz, G., Dehaene, S. \& Hertz-Pannier, L. (2002) Functional neuroimaging of speech perception in infants. Science, 298, 2013-2015.

Diamond, A. (1991) Frontal lobe involvement in cognitive changes during the first year of life. In Gibson, K.R. \& Peterson, A.C. (Eds), Brain Maturation and Cognitive Development: Comparative and Cross-Cultural Perspectives. Aldine de Gruyter, New York, pp. 127-180.

Diamond, A. (2000) Close interrelation of motor development and cognitive development and of the cerebellum and prefrontal cortex. Child Dev., 71, 44-56.

Dunbar, R. (2003) The social brain: mind, language and society in evolutionary perspective. Ann. Rev. Anthrop., 32, 163-181.

Eimer, M. \& Holmes, A. (2002) An ERP study on the time course of emotional face processing. Neuroreport, 13, 427-431.

Evans, A. (2006) The NIH study of normal brain development. Neuroimage, 30, 184-202.

Falck-Ytter, T., Gredebäck, G. \& von Hofsten, C. (2006) Infants predict other people's action goals. Nature Neurosci., 9, 878-879.

Farroni, T., Csibra, G., Simion, F. \& Johnson, M.H. (2002) Eye contact detection in humans from birth. Proc. Natl Acad. Sci. USA, 99, 9602-9605.

Farroni, T., Johnson, M.H. \& Csibra, G. (2004a) Mechanisms of eye gaze perception during infancy. J. Cogn. Neurosci., 16, 1320-1326.

Farroni, T., Pividori, D., Simion, F., Massaccesi, S. \& Johnson, M.H. (2004b) Eye gaze cueing of attention in newborns. Infancy, 5, 39-60.

Fine, C., Lumsden, J. \& Blair, R.J.R. (2001) Dissociation between 'theory of mind' and executive functions in a patient with early left amygdala damage. Brain, 124, 287-298.

Fiske, S.T. (1995) Social cognition. In Tesser, A. (Ed.), Advanced Social Psychology. .McGraw-Hill, New York, pp. 149-193.

Friedman, D. (1991) The endogenous scalp-recorded brain potentials and their relation to cognitive development. In Jennings, J.R. \& Coles, M.G.H. (Eds), Handbook of Cognitive Psychophysiology: Central and Autonomic Nervous System Approaches. John Wiley, New York, pp. 621-656.

Friedman, D., Putnam, L., Ritter, W., Hamberger, M. \& Berman, S. (1992) A developmental study of picture matching in children, adolescents, and young-adults: a replication and extension. Psychophysiology, 29, 593-610.

Frith, C.D. \& Frith, U. (1999) Interacting minds: a biological basis. Science, 286, 1692-1695.

Frith, C.D. \& Frith, U. (2006) The neural basis of mentalizing. Neuron, 50, $531-534$.

Gauthier, I. \& Nelson, C.A. (2001) The development of face expertise. Curr. Opin. Neurobiol., 11, 219-224.

Gauthier, I., Tarr, M.J., Anderson, A.W., Skudlarski, P. \& Gore, J.C. (1999) Activation of the middle fusiform 'face area' increases with expertise in recognizing novel objects. Nature Neurosci., 2, 568-573.

Grandjean, D., Sander, D., Pourtois, G., Schwartz, S., Seghier, M.L., Scherer, K.R. \& Vuilleumier, P. (2005) The voices of wrath: brain responses to angry prosody in meaningless speech. Nature Neurosci., 8, 145-146.

Grice, S.J., Halit, H., Farroni, T., Baron-Cohen, S., Bolton, P. \& Johnson, M.H. (2005) Neural correlates of eye-gaze detection in young children with autism. Cortex, 41, 342-353. 
Grossman, T., Striano, T. \& Friederici, A.D. (2007) Developmental changes in infants' processing of happy and angry facial expressions: a neurobehavioral study. Brain Cogn., in press.

Grossmann, T., Striano, T. \& Friederici, A.D. (2005) Infants' electric brain responses to emotional prosody. Neuroreport, 16, 1825-1828.

Grossmann, T., Striano, T. \& Friederici, A.D. (2006) Crossmodal integration of emotional information from face and voice in the infant brain. Dev. Sci., 9, 309-315.

de Haan, M., Belsky, J., Reid, V., Volein, A. \& Johnson, M.H. (2004) Maternal personality and infants' neural and visual responsitivity to facial expressions of emotion. J. Child Psychol. Psychiatr., 45, 1209-1218.

de Haan, M., Johnson, M.H. \& Halit, H. (2003) Development of face-sensitive event-related potentials during infancy: a review. Int. J. Psychophysiol., 51, 45-58.

de Haan, M. \& Nelson, C.A. (1997) Recognition of the mother's face by six-month old infants: a neurobehavioral study. Child Dev., 68, 187-210.

de Haan, M. \& Nelson, C.A. (1999) Brain activity differentiates face and object processing by 6-month-old infants. Dev. Psychol., 35, 1114-1121.

de Haan, M., Pascalis, O. \& Johnson, M.H. (2002) Specialization of neural mechanisms underlying face recognition in human infants. J. Cogn. Neurosci., 14, 199-209.

de Haan, M. \& Thomas, K.M. (2002) Applications of ERP and fMRI techniques to developmental science. Dev. Sci., 5, 335-343.

Halit, H., de Haan, M. \& Johnson, M.H. (2003) Cortical specialisation for face processing: face-sensitive event-related potential components in 3- and 12- month-old infants. Neuroimage, 19, 1180-1193.

Halit, H., Csibra, G., Volein, A. \& Johnson, M.H. (2004) Face-sensitive cortical processing in early infancy. J. Child Psychol. Psychiatry, 45, 1228-1234.

Haxby, J.V., Hoffman, E.A. \& Gobbini, M.I. (2000) The distributed human neural system for face perception. Trends Cogn. Sci., 4, 223-233.

Heavey, L., Phillips, W., Baron-Cohen, S. \& Rutter, M. (2000) The awkward moments test: a naturalistic measure of social understanding in autism. J. Autism Dev. Disord., 30, 225-236.

Hirai, M. \& Hiraki, K. (2005) An event-related potentials study of biological motion perception in human infants. Cognitive Brain Res., 22, 301-304.

Huttenlocher, P.R. (2002) Neural Plasticity. Harvard University Press, Cambridge, MA.

Huttenlocher, P.R. \& Dabholkar, A.S. (1997) Regional differences in synaptogenesis in human cerebral cortex. J. Comp. Neurol., 387, 167 178

Izard, C.E. (1991) The Psychology of Emotions. Plenum, London.

Johansson, G. (1973) Visual perception of biological motion and a model for its analysis. Perception Psychophysics, 14, 201-211.

Johnson, M.H. (2001) Functional brain development in humans. Nature Rev. Neurosci., 2, 475-483.

Johnson, M.H. (2005a) Developmental Cognitive Neuroscience. Blackwell, Oxford.

Johnson, M.H. (2005b) Subcortical face processing. Nature Rev. Neurosci., 6, 766-774.

Johnson, M.H., Griffin, R., Csibra, G., Halit, H., Farroni, T., de Haan, M., Baron-Cohen, S. \& Richards, J. (2005) The emergence of the social brain network: evidence from typical and atypical development. Dev. Psychopathol., 17, 599-619.

Kampe, K., Frith, C.D. \& Frith, U. (2003) 'Hey John': signals conveying communicative intention toward self activate brain regions associated with 'mentalizing', regardless of modality. J. Neurosci., 12, 5258-5263.

Kanwisher, N. (2000) Domain specificity in face processing. Nature Neurosci., 3, 759-763.

Karmiloff-Smith, A., Klima, E., Bellugi, U., Grant, J. \& Baron-Cohen, S. (1995) Is there a social module? Language, face processing, and theory of mind in individuals with Williams syndrome. J. Cogn. Neurosci., 7, 196208.

Keil, A. \& Gruber, T. \& Mueller, M. (2001) Functional correlates of macroscopic high- frequency brain activity in the human visual system. Neurosci. Biobehav. Rev., 25, 527-534.

Kimble, C.E. \& Olszewski, D.A. (1980) Gaze and emotional expression: the effects of message positivity-negativity and emotional intensity. J. Res. Pers., 14, 60-69.

Kleinke, C.L. (1986) Gaze and eye contact: a research review. Psychol. Bull., 100, 68-72.

Klin, A. (2000) Attributing social meaning to ambiguous visual stimuli in higher-functioning autism and Asperger syndrome: the social attribution task. J. Child Psychol. Psychiatry, 41, 831-846.

Kuhl, P. (2004) Early language acquisition: cracking the speech code. Nature Rev. Neurosci., 5, 831-841.
Lieberman, M.D. (2006) Social cognitive neuroscience: a review of core processes. Annu. Rev. Psychol., 58, 18.1-18.31.

Meltzoff, A.N. (2002) Imitation as a mechanism of social cognition: origins of empathy, theory of mind, and the representation of action. In Goswami, U. (Ed.), Handbook of Childhood Cognitive Development. Blackwell Publishers, Oxford, pp. 6-25.

Meltzoff, A.N. (2005) Imitation and other minds: the 'Like Me' hypothesis. In Hurley, S. \& Chater, N. (Eds), Perspectives on Imitation: from Cognitive Neuroscience to Social Science. MIT Press, Cambridge, MA, pp. 55-77.

Meltzoff, A.N. \& Decety, J. (2003) What imitation tells us about social cognition: a rapprochement between developmental psychology and cognitive neuroscience. Philos. Trans. R. Soc. London Series B, 358, 491-500.

Mitchell, R.L.C., Elliott, R., Barry, M., Cruttenden, A. \& Woodruff, P.W. R. (2003) The neural response to emotional prosody, as revealed by functional magnetic resonance imaging. Neuropsychologia, 41, 1410-1421.

Morton, J. \& Johnson, M.H. (1991) CONSPEC and CONLERN: a two-process theory of infant face recognition. Psychol. Rev., 98, 164-181.

Müller, M.M., Gruber, T. \& Keil, A. (2000) Modulation of induced gamma band activity in the human EEG by attention and visual information processing. Int. J. Psychophysiol., 38, 283-299.

Mundy, P., Card, J. \& Fox, N. (2000) Fourteen month cortical activity and different infant joint attention skills. Dev. Psychobiol., 36, 325-338.

Mundy, P., Fox, N. \& Card, J. (2003) EEG coherence, joint attention and language development in the second year. Dev. Sci., 6, 48-54.

Nelson, C.A. \& Collins, P.F. (1991) Event-related potential and looking time analysis of infants' responses to familiar and novel events: Implications for visual recognition memory. Dev. Psychol., 27, 50-58.

Nelson, C.A. \& de Haan, M. (1996) Neural correlates of infants' visual responsiveness to facial expression of emotion. Dev. Psychobiol., 29, 577 595

Nelson, C.A., Thomas, K.M., de Haan, M. \& Wewerka, S. (1998) Delayed recognition memory in infants and adults as revealed by event-related potentials. Int. J. Psychophysiol., 29, 145-165.

Nystroem, P., Rosander, K. \& von Hofsten, K. (2006) Action perception at 5 months: are infants mirroring the actor. Paper Presented at International Conference for Infant Studies, Kyoto, Japan, June 2006. [International society for Infant Studies]

Oberman, L.M., Hubbard, E.M., McCleery, J.P., Altschuler, E.L., Ramachandran, V.S. \& Pineda, J.A. (2005) EEG evidence for mirror neuron dysfunction in autism spectrum disorders. Brain Res. Cogn. Brain Res., 24, 190-198

Ochsner, K.N. \& Lieberman, M.D. (2001) The emergence of social cognitive neuroscience. Am. Psychol., 56, 717-734.

Onishi, K. \& Baillargeon, R. (2005) Do 15-month-old infants understand false beliefs? Science, 308, 255-258.

Parker, S.W., Nelson, C.A. \& the Bucharest Early Intervention Project Core Group. (2005) The impact of early institutional rearing on the ability to discriminate facial expressions of emotion: an event-related potential study. Child Dev., 76, 54-72.

Passarotti, A.M., Paul, B.M., Bussiere, J., Buxton, R., Wong, E. \& Stiles, J. (2003) Development of face and location processing: a fMRI study. Dev. Sci., 6, 100-117.

Pelphrey, K.A., Singerman, J.D., Allison, T. \& McCarthy, G. (2003) Brain activation evoked by perception of gaze shifts: the influence of context Neuropsychologia, 41, 156-170.

Posner, M.I. \& Rothbart, M.K. (2000) Developing mechanisms of self regulation. Dev. Psychopathol., 12, 427-441.

Reid, V.M., Belsky, J. \& Johnson, M.H. (2005) Infant perception of human action: Toward a developmental cognitive neuroscience of individual differences. Cogn. Brain Behav., 9, 35-52.

Reid, V.M., Csibra, G., Belsky, J. \& Johnson, M.H. (2007) Neural correlates of the perception of goal-directed action in infants. Acta Psychologica, 124, 129-138.

Reid, V.M., Hoehl, S. \& Striano, T. (2006) The perception of biological motion by infants: an event-related potential Study. Neuroscience Lett., 395, 211 214

Reid, V.M., Striano, T., Kaufman, J. \& Johnson, M. (2004) Eye gaze cuing facilitates neural processing of objects in 4 month old infants. Neuroreport, 15, 2553-2556.

Reynolds, G.D. \& Richards, J.E. (2005) Familiarization, attention, and recognition memory in infancy: an ERP and cortical source analysis study. Dev. Psychol., 41, 598-615.

Richards, J.E. (2004) Recovering dipole sources from scalp-recorded eventrelated-potentials using component analysis: principal component analysis and independent component analysis. Int. J. Psychophysiol., 54, 201-220. 
Rizzolatti, G. \& Craighero, L. (2004) The mirror-neuron system. Annu. Rev Neurosci., 27, 169-192.

Ronald, A., Happe, F. \& Plomin, R. (2005) The genetic relationship between individual differences in social and non-social behaviours characteristic of autism. Dev. Sci., 8, 444-458.

Rugg, M.D. \& Coles, M.G.H. (1995) Electrophysiology of Mind: Event-Related Brain Potentials and Cognition. Oxford University Press, Oxford.

Saxe, R. (2006) Uniquely human social cognition. Curr. Opin. Neurobiol., 16, 235-239.

Scherer, K.R. (1989) Vocal correlates of emotion. In Manstead, A. \& Wagner, H. (Eds), Handbook of Psychophysiology: Emotion and Social Behavior. Wiley, London, pp. 165-197.

Scherer, K.R., Banse, R. \& Wallbott, H.G. (2001) Emotion inferences from vocal expression correlate across languages and cultures. J. Cross-Cultural Psychol., 32, 76-92.

de Schonen, S. \& Mathivet, E. (1990) Hemispheric asymmetry in a face discrimination task in infants. Child Dev., 61, 1192-1205.

Senju, A., Johnson, M.H. \& Csibra, G. (2006) The development and neural basis of referential gaze perception. Soc. Neurosci., 1, 220-234.

Serrano, J.M., Iglesias, J. \& Loeches, A. (1995) Infants' responses to adult static facial expressions. Infant Behav. Dev., 18, 477-482.

Slotnick, S.D., Klein, S.A., Carney, T., Sutter, E.E. \& Dastmalchi, S. (1999) Using multi-stimulus VEP source localization to obtain a retinotopic map of human primary visual cortex. Clin. Neurophysiol., 110, 1793-1800.

Snyder, K., Webb, S.J. \& Nelson, C.A. (2002) Theoretical and methodological implications of variability in infant brain response during recognition memory paradigm. Infant Behav. Dev., 25, 466-494.

Soken, N.H. \& Pick, A.D. (1992) Intermodal perception of happy and angry expressive behaviors by seven-month-old infants. Child Dev., 63, 787-795.

Southgate, V., Senju, A. \& Csibra, G. (2007) Action anticipation through attribution of false belief by two-year-olds. Psychol. Sci., in press.

Spitzer, M. (2002) Lernen: Gehirnforschung und die Schule des Lebens. Spekturm Akademischer Verlag, Heidelberg.

Stern, D. (1985) The Interpersonal World of the Infant. Basic Books, New York.

Striano, T., Kopp, F., Grossmann, T. \& Reid, V.M. (2006a) Eye contact influences neural processing of emotional expressions in 4-month-old infants. Soc. Cogn. Affect. Neurosci., 1, 87-95.
Striano, T., Reid, V.M. \& Hoehl, S. (2006b) Neural mechanisms of joint attention in infancy. Eur. J. Neurosci., 23, 2819-2823.

Striano, T. \& Stahl, D. (2005) Sensitivity to triadic attention in early infancy. Dev. Sci., 4, 333-343.

Surian, L., Caldi, S. \& Sperber, D. (2007) Attribution of beliefs by 13-month-old infants. Psychol. Sci., in press.

Tallon-Baudry, C. \& Bertrand, O. (1999) Oscillatory gamma activity in humans and its role in object representation. Trends Cogn. Sci., 3, 151162.

Taylor, M.J., Batty, M. \& Itier, R.J. (2004) The faces of development: a review of early face processing over childhood. J. Cogn. Neurosci., 16, $1426-1442$.

Taylor, M.J., Itier, R.J., Allison, T. \& Edmonds, G.E. (2001) Direction of gaze effects on early face processing: eyes-only vs. full faces. Cogn. Brain Res., 10, 333-340.

Thatcher, R.W., Krause, P.J. \& Hrybyk, M. (1986) Cortico-cortical associations and EEG coherence: a two-compartmental model. Electroencephalogr. Clin. Neurophysiol., 64, 123-143.

Tomasello, M., Carpenter, M., Call, J., Behne, T. \& Moll, H. (2005) Understanding and sharing intentions: the origins of cultural cognition. Behav. Brain Sci., 28, 675-691.

Troje, N.F. \& Westhoff, C. (2006) The inversion effect in biological motion perception: evidence for a 'life detector'? Curr. Biol., 16, 821-824.

Tzourio-Mazoyer, N., De Schonen, S., Crivello, F., Reutter, B., Aujard, Y. \& Mazoyer, B. (2002) Neural correlates of woman face processing by 2-monthold infants. Neuroimage, 15, 454-461.

Walker, A.S. (1982) Intermodal perception of expressive behaviors by human infants. J. Exp. Child Psychol., 33, 514-535.

Walker-Andrews, A.S. (1986) Intermodal perception of expressive behaviors: relation of eye and voice? Dev. Psychol., 22, 373-377.

Walker-Andrews, A.S. (1997) Infants' perception of expressive behaviors: differentiation of multimodal information. Psychol. Bull., 121, 1-20.

Whalen, P.J., Shin, L.M., McInerney, S.C., Fischer, H., Wright, C.I. \& Rauch, S.L. (2001) A functional MRI study of human amygdala responses to facial expressions of fear and anger. Emotion, 1, 70-83.

Whiten, A. \& Byrne, R.W. (1997) Machiavellian Intelligence II: Evaluations and Extensions. Cambridge University Press, Cambridge. 